\title{
A Planetary Companion around a Metal-Poor Star with Extragalactic Origin
}

\author{
Johny Setiawan*, Rainer Klement*, Thomas Henning*, Hans-Walter Rix*, \\ Boyke Rochau*, Tim Schulze-Hartung* and Jens Rodmann ${ }^{\dagger}$ \\ *Max-Planck-Institut für Astronomie, Königstuhl 17, 69117 Heidelberg, Germany \\ ${ }^{\dagger}$ European Space Agency, Space Environment and Effects Section, ESTEC, Keplerlaan 1, 2201 AZ \\ Noordwijk, The Netherlands
}

\begin{abstract}
We report the detection of a planetary companion around HIP 13044, a metal-poor star on the red Horizontal Branch. The detection is based on radial velocity observations with FEROS, a high-resolution spectrograph at the 2.2-m MPG/ESO telescope, located at ESO La Silla observatory in Chile. The periodic radial velocity variation of $P=16.2$ days can be distinguished from the periods of the stellar activity indicators. We computed a minimum planetary mass of $1.25 \mathrm{M}_{\mathrm{Jup}}$ and an orbital semi-major axis of $0.116 \mathrm{AU}$ for the planet. This discovery is unique in three aspects: First, it is the first planet detection around a star with a metallicity much lower than few percent of the solar value; second, the planet host star resides in a stellar evolutionary stage that is still unexplored in the exoplanet surveys; third, the star HIP 13044 belongs to one of the most significant stellar halo streams in the solar neighborhood, implying an extragalactic origin of the planetary system HIP 13044 in a disrupted former satellite of the Milky Way.
\end{abstract}

Keywords: Planetary systems, Red Horizontal Branch Star, HIP 13044, Radial velocity PACS: 90

\section{INTRODUCTION}

Several hundred extrasolar planets beyond our Solar system have been discovered in the last two decades. So far, the majority are companions around solar-like stars. Compared to these discoveries, there is only a small number of planet detections in the late evolutionary state, like around Red Giant Branch (RGB) stars and pulsars. Even more so, the phase directly after the RGB stage, the Horizontal Branch (HB), is still unexplored.

Besides the evolutionary stage, a star's chemical composition seems to be a major indicator of its probability for hosting a planet. Early studies, e.g., [13], showed that giant planet host stars are metal-rich. This finding is supported by the large exoplanet search surveys around main-sequence stars that reported the planet-metallicity connection [29, 37]. Moreover, the survey of metal-poor main-sequence stars e.g. [35] has found no evidence for Jovian planets.

Until now, only very few planets have been discovered around stars with metallicities as low as $[\mathrm{Fe} / \mathrm{H}]=-1$, i.e. $10 \%$ of the Sun's metallicity. The discovery of PSR B1620 $\mathrm{b}$, a Jovian planet orbiting a pulsar in the core of the metal-poor globular cluster M4 $([\mathrm{Fe} / \mathrm{H}]=-1.2)$, implies, however, that planets might have formed around metal-poor stars $[9,32]$. 


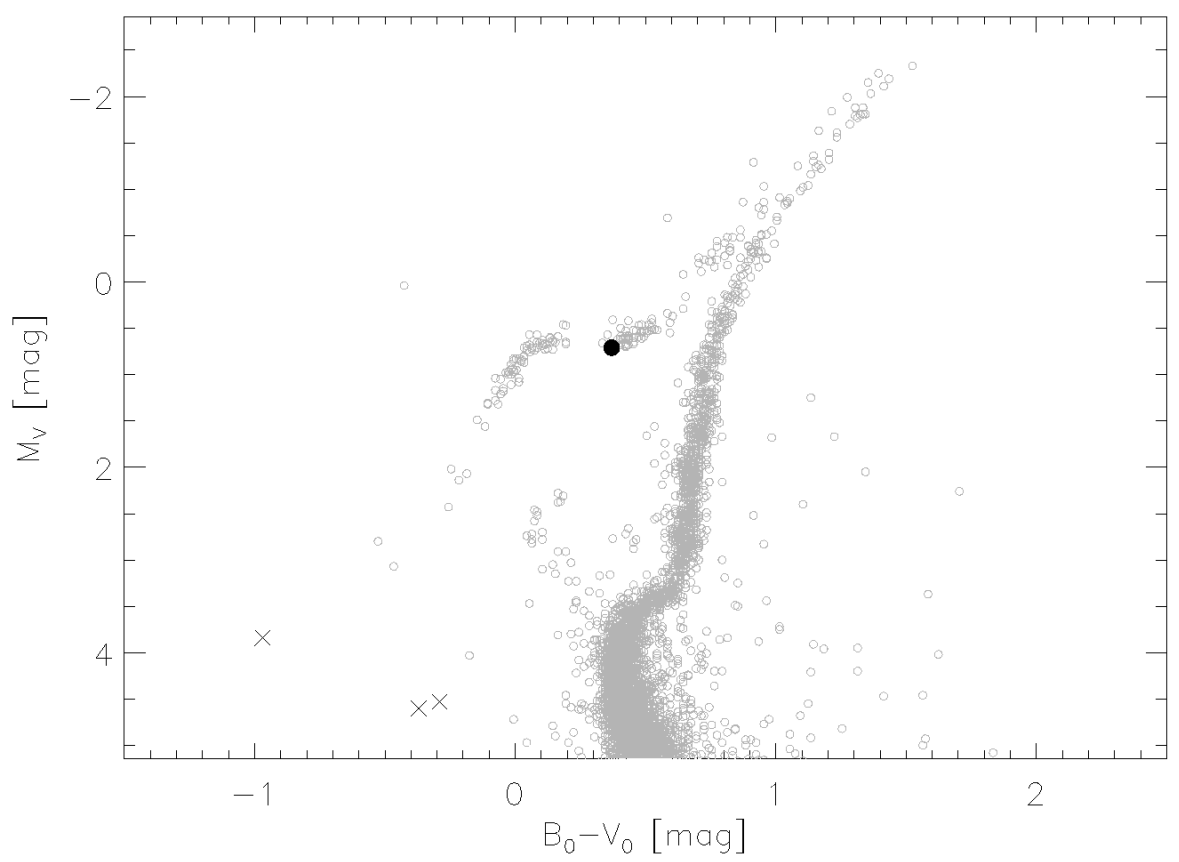

FIGURE 1. Location of HIP 13044 in a $\mathrm{M}_{V}$ vs. $B-V$ color-magnitude diagram (CMD) shown as a black dot superimposed to the CMD of Messier 3 (grey open circles) based on the photometry by [3]. Further candidates for post RGB stars hosting planets/brown dwarf, V391 Peg, HW Vir and HD 149382 $[33,22,10]$ are displayed as crosses.

TABLE 1. Stellar parameters of HIP 13044

\begin{tabular}{llll}
\hline Parameter & Value & Unit & Reference* $^{*}$ \\
\hline Spectral type & $\mathrm{F} 2$ & & SIMBAD \\
$m_{V}$ & 9.94 & $\mathrm{mag}$ & Hipparcos \\
distance & $701 \pm 20$ & $\mathrm{pc}$ & {$[1],[2],[3]$} \\
$T_{\text {eff }}$ & $6025 \pm 63$ & $\mathrm{~K}$ & {$[3],[4]$} \\
$R_{*}$ & $6.7 \pm 0.3$ & $\mathrm{R}_{\odot}$ & {$[3]$} \\
$\log g$ & $2.69 \pm 0.3$ & & {$[3]$} \\
$m$ & $0.8 \pm 0.1$ & $\mathrm{M}_{\odot}$ & this work \\
{$[F e / H]$} & $-2.09 \pm 0.26$ & {$[\mathrm{Fe} / \mathrm{H}] \odot$} & {$[1],[4],[5],[6]$} \\
$v \sin i$ & $8.8 \pm 0.8$ & $\mathrm{~km} \mathrm{~s}^{-1}$ & {$[3]$} \\
& $11.7 \pm 1.0$ & $\mathrm{~km} \mathrm{~s}^{-1}$ & this work \\
\hline
\end{tabular}

* [1]:[2];[2]:[4];[3]:[5]; [4]:[28];[5]:[7];[6]:[6]

\section{THE STAR HIP 13044}

HIP 13044 is a star of spectral type F2 (SIMBAD). It resides in the red part of the Horizontal Branch (RHB), which is separated from the blue and extreme Horizontal Branch (BHB and EHB) stars by the RR Lyrae instability strip (Fig. 1). Table 1 summarizes the fundamental stellar parameters.

The stellar mass has been inferred from the knowledge of the stellar radius $R_{*}$ and surface gravity $\log g$. We calculated a stellar mass of $0.8 \pm 0.1 M_{\odot}$. [5] has measured 
$v \sin i=8.8 \mathrm{~km} \mathrm{~s}^{-1}$, whereas we obtained $v \sin i=11.7 \mathrm{~km} \mathrm{~s}^{-1}$. The discrepancy between the two results is probably caused by the different methods used. [5] used a Fourier transform method, whereas we used a cross-correlation technique to measure the $v \sin i$ [30]. For this work we adopt $v \sin i=10.25 \pm 2.1 \mathrm{~km} \mathrm{~s}^{-1}$ which is the mean value of both measurements.

The star is known to be a member of the Helmi stellar stream, a group of stars that share similar orbital parameters that, however, stand apart from the orbits of the bulk of all the other stars. The stream stars once were bound to a satellite galaxy of the Milky Way $[16,7]$ that was tidally disrupted 6-9 Gyr ago [19]. The stream membership of HIP 13044 has been confirmed by several authors [27, 19, 28]. HIP 13044 shares the property of other stream members, like the low iron abundances $([\mathrm{Fe} / \mathrm{H}]=-1.8$ for 33 stream members) $[19,21]$ and a chemical similarity to typical inner halo stars [28]. An extensive abundance analysis of HIP 13044 has been also presented in [28].

\section{OBSERVATIONS}

Previous radial velocity (RV) measurements of HIP 13044 showed a systematic velocity of about $300 \mathrm{~km} \mathrm{~s}^{-1}$ with respect to the Sun, indicating that the star belongs to the stellar halo [4].

We observed HIP 13044 from September 2009 until July 2010 with FEROS [18], a high-resolution spectrograph $(R=48000)$ attached at the 2.2 meter Max-Planck Gesellschaft/European Southern Observatory (MPG/ESO) telescope, located at the ESO La Silla observatory in Chile. To measure the RV values of HIP 13044 we used a crosscorrelation technique, where the stellar spectrum is cross-correlated with a numerical template (mask) designed for stars of the spectral type F0.

\section{Radial velocity variation}

The variation of the RV between our observations has a semi-amplitude of $120 \mathrm{~m} \mathrm{~s}^{-1}$. In order to search for periodic variations, we used periodogram analysis techniques, which are capable of treating missing values and unevenly spaced time points. The Generalized Lomb Scargle (GLS) periodogram [39] reveals a significant RV periodicity at $P=16.2$ days with a False Alarm Probability (FAP) of $5.5 \times 10^{-6}$ (Fig. 2). Additional analysis by using a Bayesian algorithm [12] yielded also a period around 16 days.

Such RV variation can be induced in principle either by an unseen orbiting companion, by moving/rotating surface inhomogeneities or by non-radial stellar pulsations. Exploring both stellar rotational modulation and pulsations is critical when probing the presence of a planetary companion, since they can produce a similar or even the same RV variation, mimicking a Keplerian motion. 

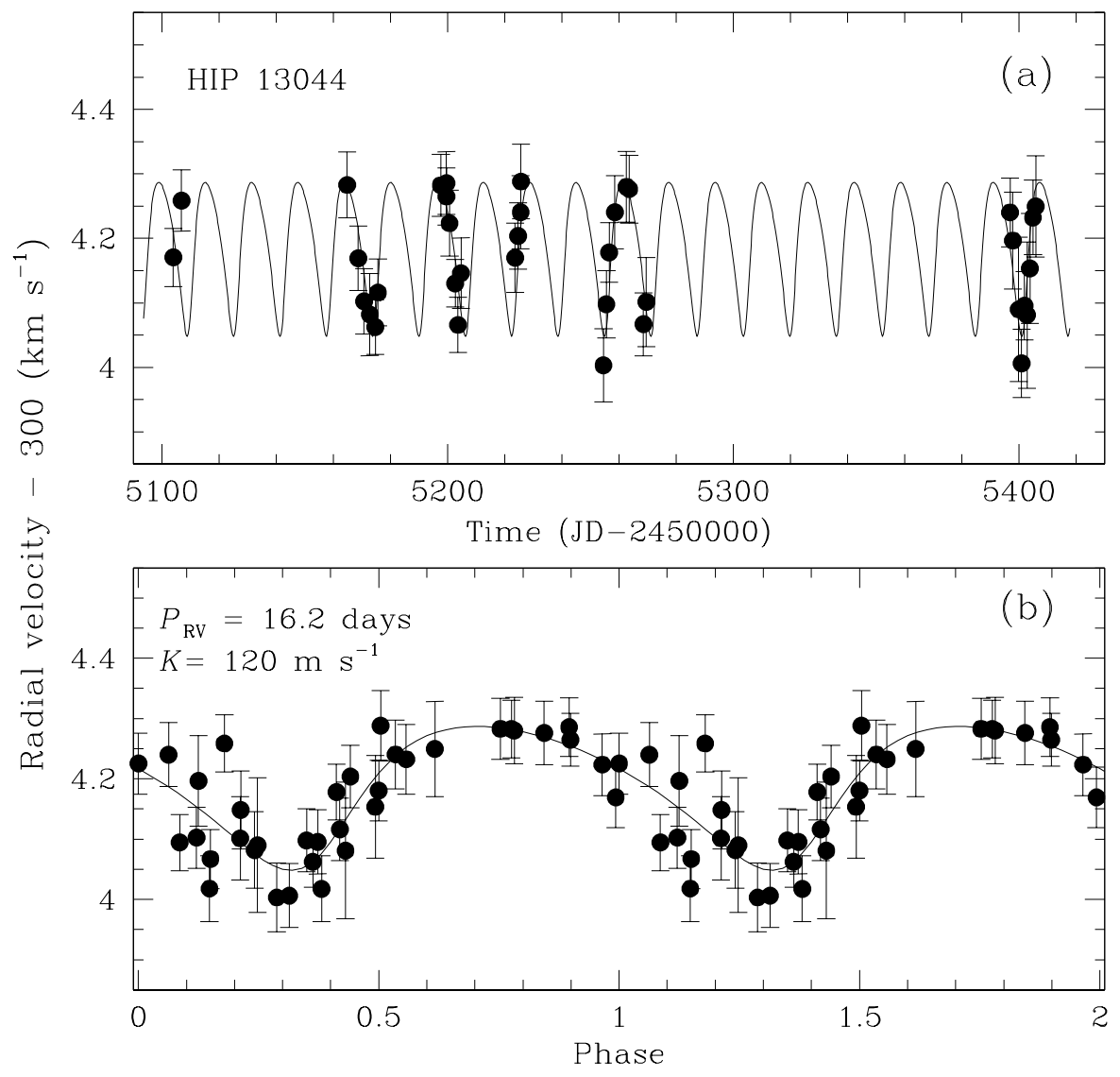

FIGURE 2. RV variation of HIP 13044 is shown in the upper panel. The lower panel shows the RV variation phase-folded with $P=16.2$ days. The amplitude of the RV variation is $120 \mathrm{~m} \mathrm{~s}^{-1}$.

\section{Stellar activity}

\section{Bisector and Ca II}

A well-established technique to detect stellar rotational modulation is to investigate the line profile asymmetry or bisector [11] and Ca II lines. Surface inhomogeneities, such as star spots and large granulation cells, will produce asymmetry in the spectral line profiles. Instead of measuring the bisectors, one can equivalently use the bisector velocity spans (BVS) to search for rotational modulation [15]. Adopting this technique, we have measured BVS from the stellar spectra. We found only a weak correlation between BVS and RV (correlation coefficient $=-0.13$ ). Interestingly, we found that the BVS variation shows a clear periodicity with $P=5.02$ days (Fig. 3 ). No period around 16 days is found in the BVS variation.

In addition to the BVS analysis, we investigated the variation of the Ca II $\lambda 849.8$ line, which is one of the Ca II infrared triplet lines. From the observed Ca II 849.8 equivalent width variations we computed a mean period of 6.05 days, which is in the same order of the period of the BVS variation. We adopt the mean period of both stellar activity 

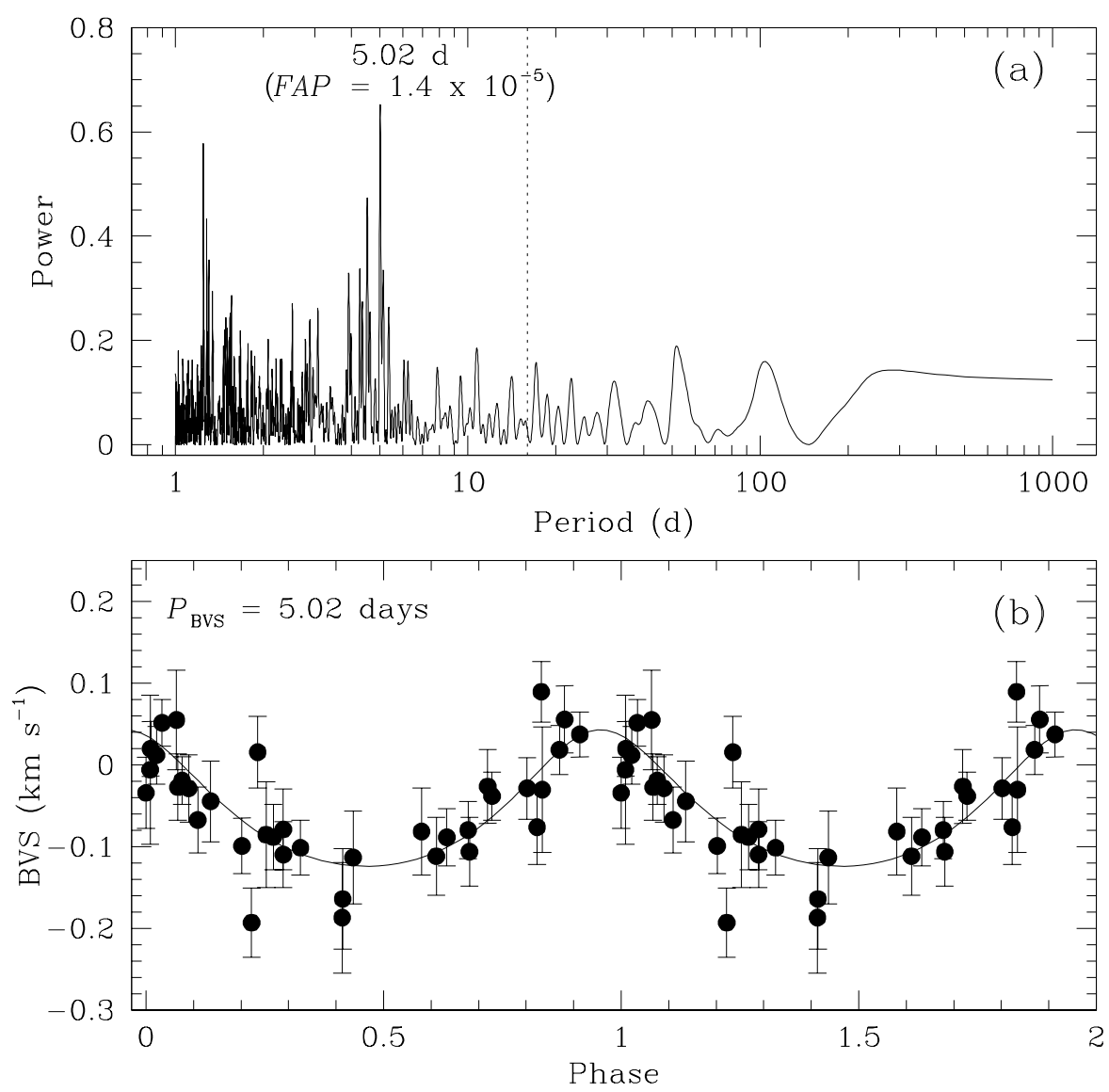

FIGURE 3. Periodogram analysis of the BVS variation showing a periodicity of 5.02 days with a FAP of $1.4 \times 10^{-5}$ (upper panel). The lower panel shows a phase-folded plot of the BVS variation. The semiamplitude of the variation is $\sim 70 \mathrm{~ms}^{-1}$.

indicators, $P_{\text {rot }}=5.53 \pm 0.73$ days, as the stellar rotation period of HIP 13044 .

\section{Photometric observations}

We found evidence for stellar pulsations of HIP 13044 from the photometric measurements with Hipparcos [25] and SuperWASP [26]. While the Hipparcos data shows only one marginal significant periodicity of 7.1 hours, the SuperWASP data shows two significant periodicities of $P_{1}=1.39$ days and $P_{2}=3.53$ days $\left(\mathrm{FAP}=5 \times 10^{-4}\right.$ and $\left.2 \times 10^{-4}\right)$. These two signals, however, are harmonic to each other $\left(1 / P_{1}+1 / P_{2}=1\right)$.

The timescale of the Hipparcos photometric variation is similar to oscillation periods found in evolved stars in the metal-poor globular cluster NGC 6397 [36]. According to the empirical relation by [20] we estimated a oscillation frequency of $53 \mu \mathrm{Hz}$ that corresponds to a period of 5.2 hours. The timescale of the derived periodicity is further consistent with theoretical models of pulsations of HB stars [38].

However, the periodicities found in the SuperWASP data do not seem to support the 
TABLE 2. Orbital parameters of HIP $13044 \mathrm{~b}$

\begin{tabular}{lll}
\hline$P$ & $16.2 \pm 0.3$ & days \\
$K_{1}$ & $119.9 \pm 9.8$ & $\mathrm{~m} \mathrm{~s}^{-1}$ \\
$e$ & $0.25 \pm 0.05$ & \\
$\omega$ & $219.8 \pm 1.8$ & deg \\
$J D_{0}-2450000$ & $5109.78 \pm 0.02$ & days \\
$\chi^{2}$ & 32.35 & $\mathrm{~m} \mathrm{~s}^{-1}$ \\
$r m s$ & 50.86 & $\mathrm{~m} \mathrm{~s}^{-1}$ \\
$m_{2} \operatorname{sini}$ & $1.25 \pm 0.05$ & $\mathrm{M}_{\text {Jup }}$ \\
$a$ & $0.116 \pm 0.01$ & $\mathrm{AU}$ \\
\hline
\end{tabular}

models by [20] and [38]. It is possible, that these are high-order overtones of the stellar pulsations. Although the true nature of the stellar oscillations of HIP 13044 is still not yet fully understood, based on the results of the SuperWASP photometric observations, stellar pulsations can be ruled out as the source of the 16.2 days periodic RV variation.

\section{PLANETARY COMPANION}

Based on the above results the best and only viable explanation for the $\sim 16$ days period is the presence of an unseen companion. We computed the orbital solution of the companion, as given in Table 2.

The minimum mass of the companion lies securely in the planetary mass domain, even with a plausible sin $i$ uncertainty. With an eccentricity of 0.25 and a semi-major axis of $0.116 \mathrm{AU}$, the planet has a periastron distance of about $0.087 \mathrm{AU}$ which is $\approx 2.8$ times of the present stellar radius. The non-circular orbit $(e=0.25)$ is, however, not expected for a close-in giant planet around a post RGB star. It is possible that there is a third body in the system that affects the planetary orbit.

In the case of HIP 13044, the original orbit could have been disturbed or changed during the evolution of the star-planet-system, in particular during the RGB phase [34]. Interestingly, the orbital period of HIP $13044 \mathrm{~b}$ is close to three times the stellar rotation period. Such planetary systems are particularly interesting to study the starplanet interaction e.g., [31].

\section{DISCUSSION}

The discovery of a planetary companion around HIP 13044 is a particularly interesting finding since the star has a very low metallicity $([\mathrm{Fe} / \mathrm{H}] \approx-2.1)$. This is the lowest stellar metallicity among all known planet host stars so far (Fig. 4).

It might be the case that initially, the star had a higher metallicity in the planet formation phase and subsequently lost the metallicity during its evolution, especially in the giant phase via complex mechanisms. Such a mechanism could be the incorporation of heavy elements into dust grains followed by their separation from the atmosphere [24]. However, given the star's membership of the Helmi stream, in which the most 


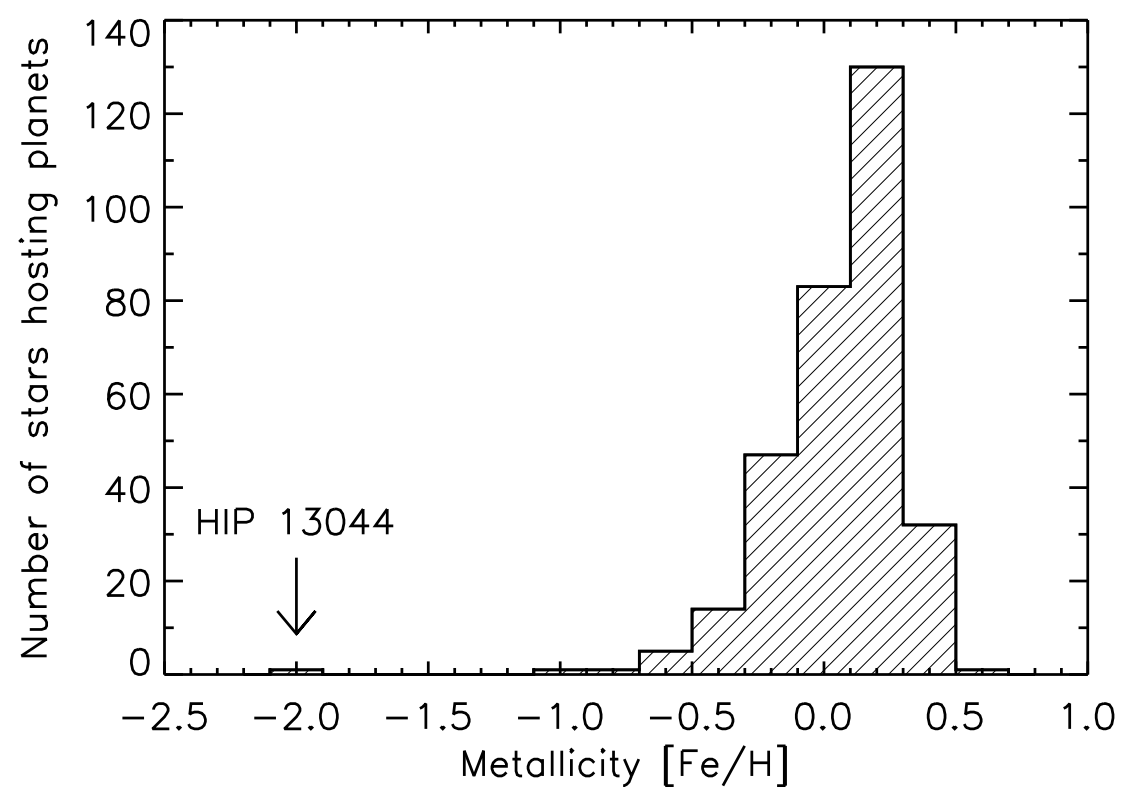

FIGURE 4. Distribution of the metallicity $[\mathrm{Fe} / \mathrm{H}]$ of planet host stars. HIP 13044, with a mean metallicity estimate of $[\mathrm{Fe} / \mathrm{H}]=-2$ is far more metal-poor than any previously known exo-planet host star.

metal-rich subdwarfs known so far have $[\mathrm{Fe} / \mathrm{H}] \sim-1.5[21]$, we do not expect its initial Fe abundance to exceed this value.

So far, there are only very few planet/brown dwarf detections around post RGB stars beyond the pulsar planets, e.g. V391 Peg [33], HW Vir [22] and HD 149382 (10, Fig. 1); see also Silvotti et al. (these proceedings) for a complete list. These are, however, substellar companions around subdwarf-B or Extreme Horizontal Branch (EHB) stars, i.e, the nature of the host stars is different from RHB stars like HIP 13044. Contrary to planet search surveys around RGB stars, such as $\mathrm{G}$ and $\mathrm{K}$ giants, that have been carried out extensively [14, 30, 8], HB stars are still unexplored.

While at least 150 main-sequence stars are known to bear close-in ( $a=0.1 \mathrm{AU})$ giant planets, so far no such planets have been reported around RGB stars. A possible explanation is that the inner planets have been engulfed by the star when the stellar atmosphere expands during the giant phase. Theoretical models $[23,34,1]$ predict that massive giant planets can survive in the RGB phase under certain circumstances. In particular, the outer planets can rapidly spiral in into closer orbits, if the star has reached its Roche lobe during the giant phase. However, for an adiabatic stellar mass loss, the planetary orbits will move out. Moreover, velocity kicks can perhaps increase eccentricity if the mass-loss is asymmetric [17].

Finally, the origin of the planetary system HIP 13044 is also of high interest. As a member of the Helmi stream, HIP 13044 most probably has an extragalactic origin. This implies that the host star would have had a quite different history than the majority of known planet host stars. HIP 13044 was probably attracted to the Milky Way several Gyr ago. Before that, it could have belonged to a satellite galaxy of the Milky Way similar to Fornax or the Sagittarius dwarf spheroidal galaxy [16]. The fascinating consequence is that HIP $13044 \mathrm{~b}$ seems to be the first detected planet with a non-Galactic origin. 


\section{REFERENCES}

1. Bear, E. \& Soker, N., arXiv:1003. 4884 (2010).

2. Beers, T. C., Kage, J. A., Preston, G. W. et al., Astron. J. 100, pp. 849-883 (1990).

3. Buonanno, R., Corsi, C. E. \& Buzzoni, A. et al., Astron. Astrophys. 290, pp. 69-103 (1994).

4. Carney, B. W. \& Latham, D. W., Astron. J. 92, pp. 60-71 (1986).

5. Carney, B. W., Latham, D. W., Stefanik, R. P. et al., Astron. J. 135, pp. 196-208 (2008).

6. Carney, B. W. ,Gray, D. F., Yong et al., Astron. J. 135, pp. 892-906 (2008).

7. Chiba, M. and Beers, T. C., Astron. J. 119, pp. 2843-2865 (2000).

8. Döllinger, M. P. and Hatzes, A. P., Pasquini, L. et al., Astron. Astrophys. 472, pp. 649-652 (2007).

9. Ford, E. B., Joshi, K. J., Rasio, F. A. et al., Astrophys. J. 528, pp. 336-350 (2000).

10. Geier, S., Edelmann, H., Heber, U. et al., Astrophys. J.l 702, pp. L96-L99 (2009).

11. Gray, D. F., Carney, B. W. \& Yong, D., Astron. J. 135, pp. $2033-2037$ (2008).

12. Gregory, P. C., Bayesian Logical Data Analysis for the Physical Sciences: A Comparative Approach with 'Mathematica' Support, Cambridge University Press, Cambridge, UK (2005).

13. Gonzalez, G., Mon. Not. R. Astr. Soc. 285, pp. 403-412 (1997).

14. Hatzes, A. P. \& Cochran, W. D., Astrophys. J.l 413, pp. 339-348 (1993).

15. Hatzes, A. P., Publ. Astr. Soc. Pacific 108, pp. 839 (1996).

16. Helmi, A., White, S. D. M., de Zeeuw, P. T. et al., Nature 402, pp. 53-55 (1999).

17. Heyl, J., Mon. Not. R. Astr. Soc. 382, pp. 915-920 (2007).

18. Kaufer, A., Stahl, O., Tubbesing, S. et al., SPIE 4008, pp. 459-466 (2000).

19. Kepley, A. A., Morrison, H. L., Helmi, A. et al., Astron. J. 134, pp. 1579-1595 (2007).

20. Kjeldsen, H. \& Bedding, T. R., Astron. Astrophys. 293, pp. 87 (1995).

21. Klement, R., Rix, H.-W., Flynn, C. et al., Astrophys. J. 698, pp. 865-894 (2009).

22. Lee, J. W., Kim, S.-L., Kim, C.-H. et al., Astron. J. 137, pp. 3181-3190 (2009).

23. Livio, M. \& Soker, N., Astron. Astrophys. 125, pp. L12-L15 (1983).

24. Mathis, J. S. \& Lamers, H. J. G. L. M., Astron. Astrophys. 259, pp. L39-L42 (1992).

25. Perryman, M. A. C., The HIPPARCOS and TYCHO catalogues. Astrometric and photometric star catalogues derived from the ESA HIPPARCOS Space Astrometry Mission, ESA SP Series, 1200 (1997).

26. Pollacco, D. L., Skillen, I., Cameron, A. C. et al., Publ. Astr. Soc. Pacific 118, pp. 1407-1418 (2006).

27. Re Fiorentin, P., Helmi, A., Lattanzi, M. G. et al., Astron. Astrophys. 439, pp. 551-558 (2005).

28. Roederer, I. U.,Sneden, C., Thompson, I. B. et al., Astrophys. J. 711, pp. 573-596 (2010).

29. Santos, N. C. Israelian, G. and Mayor, M., Astron. Astrophys. 415, pp. 1153-1166 (2004).

30. Setiawan, J., Pasquini, L., da Silva, L. et al., Astron. Astrophys. 421, pp. 241-254 (2004).

31. Shkolnik, E., Bohlender, D. A. \& Walker, G. A. H. et al., Astrophys. J. 676, pp. 628-638 (2008).

32. Sigurdsson, S.,Richer, H. B., Hansen, B. M. et al., Science 301, pp. 193-196 (2003).

33. Silvotti, R., Schuh, S., Janulis, R. et al., Nature 449, pp. 189-191 (2007).

34. Soker, N., Astron. J. 116, pp. 1308-1313 (1998).

35. Sozzetti, A., Torres, G., Latham, D. W. et al., Astrophys. J. 697, pp. 544-556 (2009).

36. Stello, D. \& Gilliland, R. L., Astrophys. J. 700, pp. 949-955 (2009).

37. Valenti, J. A. and Fischer, D. A., Astrophys. J.s 159, pp. 141-166 (2005).

38. Xiong, D. R., Cheng, Q. L. \& Deng, L., Astrophys. J. 500, pp. 449 (1998).

39. Zechmeister, M. \& Kürster, M., Astron. Astrophys. 496, pp. 577-584 (2009). 